\title{
Donación de órganos en Colombia: consideraciones sociojurídicas en torno a la Ley 1805 del $2016^{*}$
}

\author{
Organ donation in Colombia: Socio-legal considerations around \\ Law 1805 of 2016
}

Misael Tirado Acero*

Fecha de recepción: 4 de noviembre del 2019

Fecha de aprobación: 9 de diciembre del 2019

\section{RESUMEN}

La Ley 1805 del 2016 establece en Colombia modificaciones a la legislación sobre donación de órganos, con la finalidad de disminuir la diferencia existente entre la oferta y la demanda. Uno de los cambios legales introducidos es la presunción legal de donación que invierte el actual funcionamiento del registro de donantes, ya que todas las personas ahora son consideradas donantes y el registro se vuelve representativo del deseo de ser excluido de dicha presunción. Esta modificación normativa supone consideraciones legales, económicas y sociales que aquí se consideran para establecer la racionalidad jurídica, económica y social de la presunción de donación de órganos. A partir de la referida ley como unidad de análisis, se plantea un desarrollo conceptual con enfoque sociojurídico sobre la donación de órganos. Se tienen en cuenta sus aspectos sociológicos y se analiza el bloque normativo y constitucional, además

Este artículo es producto del proyecto de investigación "Las normas de origen jurisprudencial: entre la validez y la eficacia”, gestionado en la Universidad Militar Nueva Granada (Bogotá, Colombia).

Citar como Tirado Acero, M. (2020). Donación de órganos en Colombia: consideraciones sociojurídicas en torno a la Ley 1805 del 2016. Via Inveniendi et Iudicandi, 15(1), 285-315. DoI: https://doi.org/10.15332/19090528/5750

** Doctor en Sociología Jurídica e Instituciones Políticas de la Universidad Externado de Colombia. Especialista en Evaluación Social de Proyectos de la Universidad de los Andes (Colombia). Especialista en Economía de la Universidad de los Andes (Colombia). Sociólogo de la Universidad Nacional de Colombia. Investigador del Grupo Red de Estudios Sociojurídicos Comparados y Políticas Públicas (REsCyPP), línea de investigación: Política Criminal, Derecho Penal y Criminología. Docente de tiempo completo de carrera de la Facultad de Derecho de la Universidad Militar Nueva Granada (Bogotá, Colombia). Correo electrónico: misael.tirado@ unimilitar.edu.co misaeltirado@gmail.com ORCID: 0000-0003-1840-1702 
de algunas normas internacionales. Una de sus conclusiones es que la norma es racional en términos jurídico-económicos porque corrige, al menos de forma teórica, el desequilibrio entre donantes y receptores de órganos, al tiempo que ampara derechos fundamentales y prolonga de forma digna la vida del receptor, con lo cual se da cumplimiento a postulados constitucionales.

Palabras clave: cuerpo, órganos humanos, donación de órgano s, oferta y demanda de órganos humanos, presunción legal de donación.

\begin{abstract}
The Colombian Law 1805 of 2016 establishes modifications to the legislation on organ donation, in order to reduce the gap between supply and demand. One of the legal changes introduced is the legal presumption of donation that reverses the current functioning of the donor registry, since all people are now considered donors and the registry becomes representative of the desire to be excluded from that presumption. This normative modification involves legal, economic, and social considerations, which are considered here to establish the legal, economic, and social rationality of the presumption of organ donation. Based on this Law as a unit of analysis, a conceptual development with a sociolegal approach on organ donation is proposed. Its sociological aspects are also taken into account and its normative and constitutional framework is analyzed, as well as some international norms. One of its conclusions is that the norm is rational in legal-economic terms as it corrects, at least in a theoretical way, the imbalance between donors and organ recipients, while protecting fundamental rights and extending the life of the recipient in a dignifying manner, thus complying with constitutional principles.
\end{abstract}

Keywords: human body, human organs, organ donation, supply and demand of human organs, legal presumption of donation. 
Blood is that fragile scarlet tree we carry within us.

Osbert Sitwell

La mayor riqueza es la salud

VIRGILIO

\section{INTRODUCCIÓN}

El Congreso de la República, mediante la Ley 1805 del 2016, modificó el marco regulatorio de la donación de órganos en Colombia, con lo cual estableció una nueva presunción legal en el ordenamiento jurídico nacional. Ella establece que es donante de órganos toda persona que, al morir, no hubiere declarado en vida su voluntad de ser excluida del registro nacional de donantes. Este, en apariencia, se torna innecesario no solo porque toda la población está incluida en el registro de forma presunta, sino porque legalmente se establece la necesidad de crear un registro para excluir a quienes en vida manifiesten su deseo de no ser donantes de órganos, y se modifica, por lo tanto, el funcionamiento del registro existente.

La donación y el trasplante de órganos, como práctica cotidiana de la medicina, no solo implica factores inmunológicos, sino que también implica consideraciones económicas, sociológicas y jurídicas que se hacen relevantes en la medida en que la presunción de donación pone en tensión derechos fundamentales, como la libertad de cultos, el derecho a disponer del cuerpo o el derecho a la salud y la vida. A partir de estos antecedentes y teniendo en cuenta que el cambio normativo ha implicado no solo una modificación de los derechos de la persona, sino también de la funcionalidad misma de la norma que establece el registro de donantes, este artículo pretende responder a la pregunta siguiente: ¡cuál es la racionalidad jurídicoeconómica y social de la donación de órganos presunta legalmente, establecida en el artículo 3 de la Ley 1805 del 2016? 


\section{Metodología}

Este artículo desarrolla, en primer lugar, el concepto de donación de órganos, para abordar luego los aspectos sociológicos que interfieren en la donación de órganos; de este modo, se analiza el bloque normativo y constitucional en torno a la presunción de donación. Se mencionan los modelos normativos en los que se inspira la presunción de donación y se utiliza como unidad de observación la Ley 1805 del 2016 mediante un análisis con perspectiva sociojurídica, sin desatender la mención de los conceptos económicos implicados en la aplicación y estudio de la norma en cuestión.

\section{Resultados}

\section{Trasplante y donación de órganos}

In about the same degree as you are helpful, you will be happy. Karl Reiland (como aparece en Demp, 2014)

Physical ills are the taxes laid upon this wretched life; some are taxed higher, and some lower, but all pay something.

Lord Chesterfield

La donación ${ }^{1}$ de órganos implica, en términos simples, el traspaso de un órgano o tejido, bien sea de una persona viva o de un cadáver reciente ${ }^{2}$, a una persona

1 Según la RAE, "donar es traspasar a otro algo o el derecho que sobre ello se tiene". Al respecto, puede verse el desarrollo teórico del tema realizado en materia de donación de órganos por Bustamante y Villareal (2009).

2 La noción de cadáver abarca de igual forma la persona que ha sufrido muerte encefálica; esto, porque aun cuando los demás órganos funcionen de forma artificial, ha cesado en el cuerpo toda vida como la conocemos. $\mathrm{Al}$ respecto, debe recordarse que el concepto de muerte no ha sido único ni inequívoco a lo largo de la historia. Así, por ejemplo, en el siglo xvin la muerte pasó de considerarse como el último respiro de un cuerpo al proceso confirmado de su descomposición. Evolucionó posteriormente, con las nociones de flujo sanguíneo, a la ausencia de latido, así como a la finalización de la circulación y la respiración (Grudka Liral, Pontes, Schirmer y Soares, 2012; Kovacs, 2003; Oliveira y Oselka, 2008). A su vez, respecto de la muerte encefálica, esta "no coincide con la perdida de integración corporal y [...] es solo un paso que constituye una etapa de la muerte en su totalidad. Al tomarse como una decisión propia de criterio de extracción de órganos para trasplante, es necesario reconocer que dichos órganos son propios de pacientes vivos y no de muertos. El definir el término 
que, como resultado de una enfermedad, ha perdido —o visto reducida - la funcionabilidad de uno o más órganos o tejidos ${ }^{3}$. El trasplante se concibe como una forma de tratamiento que, de resultar exitosa, se considera definitiva (GómezRázuri, Ballena-López y León-Jiménez, 2016). Ella es el resultado de los avances científicos de los últimos 50 años, que han permitido curar enfermedades que antes resultaban intratables, mediante la implantación de un injerto que permite al resto de un organismo conservar su funcionabilidad (Solar, Ovalle, Simian, Escobar y Beca, junio del 2008).

Esta técnica, desarrollada en el marco de las revoluciones técnico-científicas, se hace factible, según Álvarez (mayo-agosto 2004) con el descubrimiento de la histocompatibilidad en 1952. Esta posibilitó realizar el primer trasplante renal exitoso en $1954^{[4]}$, gracias al entendimiento de la individualidad genética derivada de este descubrimiento, logro que no hubiese sido posible sin los desarrollos contemporáneos de la inmunosupresión 5 .

El déficit de donantes de órganos es evidente: hay un desequilibrio entre la oferta y la demanda y, aun así, no todos los donantes resultan útiles. Existen unas condiciones mínimas exigibles en términos médico-científicos para aceptar la donación de un sujeto determinado; por ejemplo: la sanidad física del donante, su estabilidad psicológica, la voluntariedad de la acción de donación y una fuerte motivación familiar (Álvarez Hidalgo, mayo-agosto 2004); por lo tanto, con la finalidad de determinar estos requisitos, debe realizarse todo un proceso de selección que

'muerte' este no debe ser manipulado por conflictos de intereses que en deducción se ve representado en una extracción de componentes anatómicos destinados a donación para salvar la vida de diferentes pacientes” (Tirado Acero, M. 2011 a, p. 13).

3 Según Gómez et al. (2016), algunas de las enfermedades que derivan en la necesidad de un trasplante son la cirrosis hepática, atresia de vía biliares, epoc y fibrosis quística, entre otras (Alonso-Pulpón et al., 1995; Barriga, Baeza, Pereira, Besa, Caldumbide y Medel, 1999; Prieto et al., 2003).

4 Lo ejecutó Joseph Murria en el Hospital de Boston (Álvarez Hidalgo, mayo-agosto 2004).

5 La donación de órganos es concebida para algunos como un milagro de la medicina, y para otros como una forma de atentar contra consideraciones de orden divino o religioso [...][es prudente] seńalar que solo desde el momento en que aparece en el universo de las creencias médicas el trasplante como importante herramienta para la prolongación y el mejoramiento de la vida humana, las partes anatómicas se convierten en importantes repuestos para salvar vidas, encontrando así un grado de cotización dentro del mercado" (Farfán, 2006, p. 33). 
comienza con la identificación de un potencial donante; continúa con la procuración del buen estado de órganos y tejidos hasta su ablación; luego, el transporte en condiciones especiales y tiempo limitado y, finalmente, su implantación. La condición del donante debe cumplir especificaciones: diagnósticos y certificación de muerte encefálica (como estructural irreversible, ausencia de reflejos del tronco encefálico, ausencia de respiración espontánea); estudios serológicos para validación del donante (perfil de hepatitis viral, TORCH, VIH, HTLV, treponema y tripanosoma), y pruebas específicas para validación de órganos (úrea, creatinina, ecografía, perfil hepático, glicemia, etc.). Además, la tipificación de HLA (antígeno humano de histocompatibilidad) para estudio de compatibilidad donante-receptor. (Gómez-Rázuri et al., 2016)

América Latina tiene actualmente unas tasas excesivamente bajas de donación de órganos frente a las de otros países; por ejemplo, España ${ }^{6}$ tiene una tasa de 33-35 donantes p.m.p. (personas por millón de población), mientras que el promedio latinoamericano está en 8,8 p. m.p. (Gómez-Rázuri et al., 2016). Colombia tuvo en el 2014 un promedio de 20,4 p.m. p. ${ }^{7}$ (Dirección de Medicamentos y Tecnologías en Salud del Ministerio de Salud, 2015), cifra que aumentó a 22,7 p. m. p. en el 2015 (Instituto Nacional en Salud: Red de Trasplantes, 2016).

Colombia, según las cifras del Ministerio de Salud, tiene una tendencia al alza en la donación de órganos, así como en la aceptación por parte de los familiares del difunto frente a la donación, que se ubicaba cerca del $61,5 \%$. A la fecha, hay un total de 345 donantes reales frente a los 329 del 2013, y Medellín tiene el mayor número, con 139; es decir, 40,3\% del total, cifra que tuvo un cambio porcentual negativo del 6,71\% (Dirección de Medicamentos, 2015). Afirma la Red de Trasplantes que en el

6 Este país detenta el índice mundial más alto de donación de órganos desde 1991 (Caballer Miedes y Martínez Sancho, 2000) y tiene, con Austria, "un modelo de consentimiento presunto, universal y absoluto" (Zúñiga, 2015). Cabe aquí preguntarse sobre el principio de la autonomía, que se constituye en uno de los principios básicos de la bioética respecto del tema de voluntad y la autodeterminación de la persona (Guerra, Tirado y Márquez, 2012; Tirado, 2011a).

7 Según el Informe ejecutivo de donación y trasplantes de Colombia (2015), elaborado por el Instituto Nacional de Salud, la cifra de trasplantes en el 2014 fue de 19,3 p. m. p., con un total de 921 trasplantes hasta noviembre de ese año. 
2015 estaban en lista de espera 2162 personas, cifra que representa una disminución frente al 2014, con 2179 personas en espera (véase la tabla 1).

Tabla 1. Personas en lista de espera para trasplante

\begin{tabular}{|lcc|}
\hline Componente anatómico & $\begin{array}{c}\text { Estado de lista de espera al } \\
\text { 31 de diciembre del 2014 }\end{array}$ & $\begin{array}{c}\text { Estado de lista de espera al } \\
\text { 31 de diciembre del 2015 }\end{array}$ \\
\hline Riñón & 1991 & 2015 \\
\hline Hígado & 118 & 82 \\
\hline Corazón & 27 & 20 \\
\hline Pulmón & 16 & 14 \\
\hline Páncreas & & 6 \\
\hline Riñón, páncreas & 14 & 11 \\
\hline Intestino & 1 & 3 \\
\hline Corazón, riñón & 2 & 1 \\
\hline Rińón, hígado & 10 & 9 \\
\hline Hígado, rińón, páncreas & 0 & 1 \\
\hline Total & 2179 & $2162^{8}$ \\
\hline
\end{tabular}

Fuente: Instituto Nacional en Salud: Red de trasplantes, 2016, p. 3.

El Ministerio de Salud (2016) a través de su campaña "Déjalo conversado" señala que existen en Colombia aproximadamente 2029 personas a la espera de un trasplante de órganos; así mismo, durante el 2015 se hicieron 1204 procesos de trasplantes (incremento del 14\% frente al 2014) y hubo un total de 405 donantes reales, con un incremento del $17 \%$ frente al ańo anterior. Respecto del consentimiento familiar, $60 \%$ de las familias entrevistadas manifestaron su aceptación, y hubiera sido posible salvar aproximadamente 500 vidas más si el $40 \%$ restante hubiese aceptado la realización del trasplante.

Moreno y Rivera (2015) dicen que la baja tasa de donación de órganos en Latinoamérica, particularmente en México, obedece a "los mitos creados y la falta de

8 El Ministerio de Salud y Protección Social (2016) dijo que la cifra real de pacientes a la espera de recibir un trasplante en Colombia al 31 de diciembre de 2015 era de 2029 personas. 
información sobre el tema", lo que ha "condicionado que más de cien mil potenciales beneficiarios vean alejada la posibilidad de mejorar su calidad de vida” (p. 762) ${ }^{9}$. No obstante, lo enunciado hasta ahora, Solar et al. (junio del 2008) consideran, al analizar diversos autores, que

la escasez de órganos se debería a la incapacidad de convertir a los potenciales donantes en donantes efectivos y no a la falta real de donantes (Álvarez, 2007; Gracia, 2001; Mateasanz y Miranda, 2002; Palacios, 2002; Sheehy et al., 2003). Simulaciones estadísticas permiten saber que en Estados Unidos el $42 \%$ de los donantes potenciales se convierten en donantes efectivos (Sheehy et al., 2003) y que entre el 10 y el $14 \%$ no son viables por inestabilidad hemodinámica o sepsis no controlada. (Mateasanz y Miranda, 2002) ${ }^{10}$

\section{Factores que inciden en la donación}

The first and highest law must be the love of man to man. Homo homini Deus est - this is the supreme practical maxim, this is the turning point of the world's History. Feuerbach (1881)

La esencia humana no es algo abstracto e inherente a cada individuo, es en realidad, el conjunto de las relaciones sociales. Marx, Tesis sobre Feuerbach, s. f.

9 Cantarovich (2004) dice que la educación cumple un papel fundamental en el proceso de derrumbamiento de estos mitos y en la posibilidad de incrementar la donación de órganos, sin hacerla obligatoria.

10 Como conclusión de un estudio realizado entre los estudiantes y practicantes de ciencias de la salud, se adelantó "como estrategia para contrarrestar la baja provisión de órganos se han llevado a cabo múltiples investigaciones en procura de valorar la actitud y el nivel de conocimiento sobre la donación de órganos y trasplantes. Con respecto a la actitud hacia la donación por parte del público general, Spital encontró que el $63 \%$ de los encuestados opinó que donaría sus órganos, pero solo un $50 \%$ donaría los de un familiar. En este estudio, la aceptación para donar los órganos propios y los de un familiar fue bastante similar $(90,8$ y 86,2\%, respectivamente)" (Padilla-Cuadra, Mora-Chacón, Monge-Fallas y Rodríguez-Barquero, 2015, p. 181). Con respecto a este tema, véase Jung (2013), Weber y Canbay (1999), Erdogan et al. (2002), Reddy, Guleri, Khazanchi et al. (2003) y Ríos et al. (2006). 
Son múltiples los factores que inciden en la donación de órganos; no obstante, es posible dividirlos en tres grandes paradigmas: (1) el jurídico, según el cual es dable discutir no solo el cambio normativo, sino el problema de la obligatoriedad y la vulneración que esta acarrea a los derechos de terceros (Cortes, 2016); (2) las consideraciones económicas de las políticas y regulaciones de donación de órganos que buscan maximizar de forma eficiente el proceso de donación, cubriendo la demanda mediante la generación de una mayor oferta ${ }^{11}$ y (3) el factor sociológico, afectado por las consideraciones del cuerpo como construcción social ${ }^{12}$ y sus implicaciones religiosas, lo que determina la perspectiva sociojurídica como enfoque del presente análisis normativo.

Los paradigmas referidos conllevan la necesidad de un acercamiento, mediante la teoría de la representación social, concebida por Grudka Liral, Pontes, Schirmer y Soares (2012) como una importante herramienta en el proceso de entendimiento de la construcción de la realidad. Esta teoría asume que tanto "los individuos como los grupos construyen activamente la representación de los objetos sociales, con base en múltiples creencias que constantemente circulan por la sociedad (Moloney y Walker, 2002; Santos y Almeida, 2007)". Estos sistemas de creencias modifican las actitudes y decisiones en un marco de entendimiento dinámico basado en las interacciones del individuo con la sociedad; al respecto, afirma Max Weber (2012) que la religión, como sistema ordenado de creencias, desempeña un papel fundamental en la representación de la realidad que el individuo construye para sí, modificando y determinando tanto sus intereses como las acciones que realiza con la finalidad de obtener determinado resultado.

Es posible afirmar que el cuerpo ${ }^{13}$ no es más que una construcción social susceptible de ser apreciada más allá de lo biológico, según una sociología de las influencias sociales que lo afectan por las interacciones de los individuos, en diversos contextos,

11 La economía, al contrario de la creencia popular, no se trata de la administración de las divisas o las inversiones, sino que su objetivo fundamental es la asignación de los recursos escasos; en este caso, los órganos (Posner, 2013).

12 "Pensar el cuerpo en las ciencias sociales ha significado establecer como aquello que parecía individual y natural estar atravesado por las sociedades y por configuraciones históricas particulare”. (Sabido, 2011)

13 Le Breton dice que "del cuerpo nacen y se propagan las significaciones que constituyen la base de la existencia individual y colectiva" (2008, p. 7). 
que orientan o modifican sus acciones en entornos determinados (Giddens, 2000) ${ }^{14}$. El cuerpo, entendido como objeto de estudio de la sociología, es visto por Mauss (1979) como un lugar privilegiado para apreciar las costumbres que permiten entender su uso cotidiano, porque todo gesto y movimiento es la explicitación de un constructo social (Koury, Scribeno y Tijoux, 2011).

Esta construcción teórica tiene su origen en Marx (1991), para quien el hombre se produce a sí mismo mediante el hecho autogenerador del trabajo; mas no es este cualquier tipo de trabajo, sino aquel que libera dignificando para la construcción propia y no el amasamiento de capital de otro (Marx, 1976). A partir de la teoría marxista, se construyen los dos grandes paradigmas sociológicos de la configuración del cuerpo: por un lado, el cuerpo dócil y normado de Foucault y, por el otro, el cuerpo como habitus de Pierre Bourdieu (Barrera Sánchez, 2011).

Foucault $(1975 ; 1990 ; 1992)$ considera que el cuerpo es sujeto del ejercicio del poder que se construye mediante adiestramiento y disciplina de la fábrica como institución. Este poder se ejerce por la dominación, en Foucault, como forma de alcanzar la docilidad del individuo. Al respecto, afirma en la Histoire de la sexualité que el biopoder pone al cuerpo en la contabilidad de la regulación de la natalidad, la mortalidad, la fecundidad y la capacidad reproductiva, construyendo y controlando de esta manera los patrones de comportamiento, costumbre e identidad (Foucault, 1976 ; 1992).

Por su parte, Bourdieu, tal como se cita en Barrera Sánchez (2011, p. 129), afirma que "las propiedades corporales, en tanto productos sociales son aprehendidas a través de categorías de percepción y sistemas sociales de clasificación que son independientes de la distribución de las propiedades entre las clases sociales: las taxonomías al uso tienden a oponer jerarquizándola, propiedades más frecuentes entre los que dominan (es decir, las más raras) y las más frecuentes entre los dominados” (1986, p. 86). Según su teoría, se construye lo social mediante dos factores o elementos duales: por un

14 "Lo que la gente sabe de su cuerpo es lo que le han enseńado en la escuela, lo que ha visto o leído, lo que ha compartido con otros, lo que los medios de comunicación le trasmiten. En el saber popular el cuerpo no está disociado del hombre, como ocurre en la biomedicina, "en las tradiciones el cuerpo está unido al mundo" (Sodi, 2003, p. 32. citado por Carreño Dueñas, Restrepo Restrepo y Becerra (2016). 
lado, el campo (elemento objetivo), que se constituye de estructuras independientes de la conciencia y la voluntad de los agentes, y el habitus (elemento subjetivo), entendido como compendios de percepciones, pensamiento de acción que compone socialmente nuestra objetividad. Así, las dinámicas sociales se enmarcan en el campo como escenario de juego de las posiciones relativas que ocupan los distintos grupos y las relaciones entre estas, comprendiendo al tiempo la formación de la subjetividad por el habitus (Bourdieu, 1991) ${ }^{15}$.

De esta manera, la concepción religiosa sobre el cuerpo, al menos según la creencia particular de los individuos que la practican, se basa en el “... esquema de percepciones y categorizaciones con que asimos a la realidad, es el fruto de la imposición que despliegan las estructuras objetivas sobre la subjetividad" (Barrera Sánchez, 2011, p. 128); es decir, el habitus entendido como

el sistema de disposiciones duraderas y transferibles (que funcionan) como principios generadores y organizadores de prácticas y representaciones que pueden estar objetivamente adaptadas a su fin sin suponer la búsqueda consciente de fines y el dominio expreso de las operaciones necesarias para alcanzarlos [...] sin ser producto de obediencia a reglas. (Bourdieu, 1991, citado por Barrera Sánchez, 2011, p. 128)

El cuerpo es una unidad funcional y, como tal, se construye en el imaginario popular a través de diversos factores, particularmente según la subjetividad de sus interacciones sociales, escenario y relaciones, mediante las cuales se ha construido el conocimiento en torno a la donación de órganos ${ }^{16}$, compuesto en su mayoría por

15 El habitus no es más que "un esquema de percepción y categorización con el que asimos la realidad, es el fruto de la imposición que despliegan las estructuras objetivas sobre las subjetivas. La composición de los habitus esta ligada a la posición dominada por el agente en el espacio social o en los diferentes campos en los que participa. El habitus es una recopilación de relaciones incorporadas a los agentes, el cual, a su vez, conforma un sistema de relaciones con el campo. Por otra parte, el campo está compuesto por una estructura de relaciones, es una construcción histórica y social. Las posiciones ocupadas por los diferentes agentes en los campos poseen una dimensión histórica, es decir, son el producto de luchas históricas, que a su vez, se encuentran inscritas en los cuerpos y son parte constitutiva del habitus de los agentes” (Barrera Sánchez, 2011, p. 128).

16 Grudka Liral et al. (2012, p.143) exponen que "la forma en que se construyó el conocimiento de la donación de órganos por la sociedad es crucial para determinar cómo se representa a sí misma. Los primeros experimentos con trasplantes ocurrieron a finales de los 60 y fueron ampliamente difundidos en el campo del conocimiento médico y publicitados por los medios". 
procesos de mitificación a través de los medios de comunicación ${ }^{17}$. A este respecto, algunas de las causas alegadas por los familiares de potenciales donantes para negarse a la citación, según lo encontrado por Grudka et al. (2012), son la inviolabilidad del cuerpo (el temor de que se desfigure o mutile al difunto para obtener los órganos), creencia en la posibilidad de retorno a la vida ${ }^{18}$, falta de credibilidad en el sistema o la consideración de que el criterio de asignación de los órganos es netamente financiero.

Dado este aspecto de los medios de transmisión, es facil entender la representación de la inviolabilidad del cuerpo expresada por [las personas], especialmente, en el marco de una sociedad que idolatra el cuerpo. El cuerpo es el medio concreto de comunicación social: representa la individualidad, expresión inherente del ser en el mundo, es un registro de la historia de cada uno (Freitas, 2008). Con la llegada de los trasplantes, la forma en que la vida y los cuerpos eran conceptualizadas fue modificada. La muerte viene a representar la posibilidad, por donación, de salvar las vidas o mejorar la supervivencia de los pacientes con con fallos crónicos, representando [así] un nuevo paradigma del valor después de la muerte. (Roza, García, Barbosa, Mendes y Schirmer, 2010, citados por Grudka et al., 2012, pp. 143-144)

Lo anterior implica que los mitos existentes en torno a la donación de órganos como resultado de la aplicación y aproximación del habitus, en un campo de la no educación y la religión ${ }^{19}$, no son más que una manifestación social como producto del desconocimiento de las realidades médicas palpables y los procesos de las relaciones de poder. Al respecto, menciona Castro que "la verdad desprovista de todo contenido ontológico-objetivo, no es otra cosa que un efecto, un producto, deberíamos decir, de las estrategias sociales, de las relaciones de poder" (Castro, 1995, p. 117, citado

17 "Los medios masivos de comunicación tienen un gran poder en la diseminación del conocimiento científico al público, actuando como mediador del conocimiento científico y la sociedad. Frecuentemente, los medios son responsables de la comunicación de la nueva información a los individuos, definiendo así el foco del proceso discursivo en la sociedad" (Moloney y Walker, 2000, citado por Grudka et al., 2012, p. 143).

18 No solo en sentido religioso, sino también en el caso particular de la muerte cerebral porque los familiares conservan en esta situación la esperanza de que su familiar regrese mientras el corazón aún lata.

19 Según Grudka et al. (2012), al abordar el trabajo de Mosvocici (2007), hoy estas concepciones no encuentran sustento en las religiones, como resultado de la expansión de los valores altruistas de la religión, así como de la reconstrucción de la representación porque esta es una construcción histórica por naturaleza e influencia en el desarrollo del pensamiento de los individuos sobre los conceptos y la forma de proyectar la imagen. 
por Sossa Rojas, 2012). Esto se debe a que la religión disocia las normas morales propuestas y la visión de los fieles, situación que se complejiza porque

las creencias religiosas o metafísicas juegan un rol esencial en la posición ante temas morales para quienes adhieren a ellas. En este caso las grandes religiones exhortan a sus fieles a la donación de órganos apelando a la generosidad y amor al prójimo. Sin embargo, paradójicamente, muchos fieles desconocen este criterio o interpretan de forma errónea las enseñanzas morales rechazando la donación. (Rumsey, Hurdford, Cole, Rojas y Serani, 2003, citados por Solar et al., junio 2008, p. 264)

En este sentido, menciona Farfán (2006), citado por Tirado Acero (2011) en su artículo "¿Por qué en Colombia no está generalizada la práctica de la donación de órganos?", que

lo que legitima el acto de donación o veta de órganos de un cadáver por parte de un deudo es la posibilidad de salvar una vida humana. La moral religiosa, que se opone a este tipo de transacciones no puede estar por encima del derecho que tienen los hombres a la preservación de la vida. (p. 167)

El pueblo judío ${ }^{20}$, Según Grudka et al. (2012), por ejemplo, se ha opuesto tradicionalmente a cualquier utilización del cuerpo más allá de la muerte porque lo consideran una adulteración de la imagen de Dios. Igualmente, los protestantes se opusieron a la idea porque consideraban que, para alcanzar la resurrección, era necesario que el cuerpo permaneciese intacto ${ }^{21}$; no obstante, dicha posición ha mutado y la Iglesia anglicana considera hoy que el concepto de muerte cerebral es válido y que debe permitirse la utilización de los órganos sin que exista conflicto ético, siempre y cuando se haga con espíritu altruista y sin intención de lucro (Reyes Acevedo, 2005).

20 Existe en apariencia una actitud favorable en cuanto a la función del trasplante en este grupo; no obstante, permanece vigente la prohibición de beneficiarse de un cadáver, lo que pone en el limbo el criterio del judaísmo frente a la utilización de órganos de un donante cadavérico.

$21 \mathrm{Al}$ respecto, véase Rumsey et al. (2003). Influence of knowledge and religiousness on attitudes toward organ donation. Transplantation Proc., 35(8): 2845-2850. 
La sociedad japonesa veía el cadáver como un objeto impuro, según las bases del sintoísmo, lo que acarreaba implicaciones al momento de extraer los órganos, particularmente por parte de los ritos funerarios (Namihira, 1990; Reyes Acevedo, 2005). No obstante, existen algunas religiones que ven con ojos favorables la donación de órganos; por ejemplo, el budismo: a raíz de su concepción sobre la separación entre cuerpo y alma, ve esta práctica como un acto de generosidad compasiva que permite satisfacer la vida en sociedad y acercarse a la consecución del nirvana. Situación similar se presenta en el hinduismo, según el cual, mediante la aceptación del alma tras la destrucción del cuerpo, se percibe una permisión hacia la donación y disposición de los órganos en pacientes con muerte cerebral (Sugunasiri, 1990; Reyes Acevedo, 2005). Finalmente, el Islam tiene una actitud favorable hacia la donación como consecuencia de las cualidades de altruismo, generosidad, deber y cooperación presentes en el Corán (Reyes Acevedo, 2005).

\section{Normas y presunción de donación}

Si el problema de los equilibrios mal establecidos de los siglos anteriores consistía en producir lo necesario para cubrir las necesidades, el nuevo problema consiste en crear las necesidades para poder consumir lo que se produce. Salvat, M. 1975.

El marco regulatorio de la donación de órganos tiene como presupuesto esencial el derecho a la vida, consagrado constitucionalmente en el artículo $11^{[22]}$ y el bloque de convencionalidad ${ }^{23}$; esto porque la existencia humana es fuente necesaria para el ejercicio de cualquier prerrogativa, facultad o poder (Moron Díaz, 1998) ${ }^{24}$ y la dignidad le es inherente. Esta práctica también está limitada por los derechos sobre

22 "El derecho a la vida es inviolable. No habrá pena de muerte."

23 Al respecto, véase, entre otros, el artículo 1 de la Declaración Americana de Derechos de Bogotá, de 1948, así como al artículo 13 de la Declaración Universal de los Derechos Humanos de la onu, de 1948.

24 No es cualquier forma de vida la que sirve como presupuesto escencial al ejercicio de los derechos, sino que aquella que se vive dignamente es la que permite concretarlos. Al respecto, menciona la Corte Constitucional: "El concepto de vida que la Constitución consagra no corresponde simplemente al aspecto biológico, que supondría apenas la conservación de los signos vitales, sino que implica una cualificación necesaria: la vida que el Estado debe presevar exige condiciones dignas. De poco o nada sirve a la persona mantener la subsistencia si ella no responde al mínimo que configura un ser humano como tal” (Hernandez Galindo, 1994). 
el cuerpo, que son, en principio, derechos de la personalidad (nunca reales), lo que se manifiesta en la prohibición constitucional de afectar la integridad corporal, la punibilidad de los atentados contra la integridad, la prohibición de la tortura, la imposibilidad de disponer del cuerpo de forma permanente y lesionante, así como la posibilidad de establecer la forma en que se dispondrá del cadáver ${ }^{25}$ (Valencia Zea y Ortiz Monsalve, 2011).

El marco normativo internacional regula la donación de órganos mediante la directriz 3 de la oms que establece: "Un órgano solo puede ser removido del cuerpo de un dador vivo adulto ${ }^{26}$, con propósito de trasplante, si el donante otorga su consentimiento libre" (Farfán, 2006, p. 36) 27. Situación diferente aplica para el donante cadavérico, pues depende de la legislación y de la regulación que esta determine para el cadáver y su disposición. Farfán dice que "en algunas legislaciones como la cubana o la sovietica, se acepta el sistema de la socialización del cadáver, de manera que el cuerpo de la persona fallecida tiene como único titular a la sociedad" (2006, p. 68), mientras que, en otras, como la colombiana, el cadáver aún preserva algunos derechos como resultado de la personalidad, sin que exista derecho de posesión alguno sobre aquel por parte de sus herederos (Cifuentes Muñoz, 1994) ${ }^{28}$.

25 Debe entenderse que el cadáver no es un objeto susceptible de que dispongan de él sus deudos; al respecto, establece Lehmarm que "el cadáver no es cosa, cuya propiedad pueda transmitirse a los herederos, sino un residuo de la personalidad" (Lehmann, 1949).

26 Esta disposición es únicamente aplicable a los órganos simétricos frente a los cuales es posible retirar uno sin alterar sustancialmente la calidad de vida del donante.

27 "Cualquier beneficio o contraprestación que se ofrezca a la persona que cede el órgano limita sus posibilidades discernimiento, intención y libertad para otorgar el consentimiento informado" (Farfán, 2006, p. 39).

28 "Las normativas sobre trasplante de órganos pueden clasificarse en función del carácter, la condición y el ejercicio del consentimiento del donante. De este modo, es posible identificar distintos modelos de trasplante en atención, primero, a si es necesario o no que conste la voluntad expresa del donante del órgano. Si lo es, se trata de un modelo de donación 'explícita' o 'opting in'; de lo contrario, si se asume que todas las personas son potenciales donantes a menos que señalen lo contrario, estamos ante un modelo de 'consentimiento presunto' u 'opting out'. Si no existe condición para ser beneficiario de una donación de órganos, estamos ante el modelo de donación 'universal'. Si, en cambio, la voluntad de donar se convierte en un requisito para ser receptor de un órgano (es decir, si a los individuos nos es exigible la reciprocidad), el modelo será 'condicionado'. Este es uno de los cambios que han sido introducidos por la nueva ley chilena y que solo existía desde 1986 en Singapur y, desde el 2010, en Israel” (Zúńiga, 2015, p. 1331). 
La donación de órganos en Colombia estaba regulada hasta la aparición de la Ley 1805 del 2016 y, aún hoy, de forma parcial, por la Ley 9 de 1979 en sus artículos 515 a 547, adicionada por la Ley 73 de 1988 y reglamentada por los decretos 1546 de 1998 y 2493 del 2004 (Valencia Zea y Ortiz Monsalve, 2011). Este marco normativo establecía que "solo se podrá proceder a la utilización de los órganos, componentes anatómicos y líquidos orgánicos a que se refiere este artículo, cuando exista consentimiento del donante, del receptor, de los deudos, abandono del cadáver o presunción legal de donación” (artículo 1, Ley 73). La situación jurídica que más o menos permanece sin modificación es la Ley 1805 del 2016, excepto por la inclusión de los tejidos como componente orgánico y la necesidad de un consentimiento libre, previo e informado; se elimina de la ecuación la individualización del consentimiento y el abandono del cadáver ${ }^{29}$.

La legislación previa, al igual que la Ley 1805 del 2016, establecía la presunción de donación al momento de la muerte. No obstante, admitía la legislación anterior dicha presunción de donación si y solo si "dentro de las seis (6) horas siguientes a la ocurrencia de la muerte cerebral o antes de la iniciación de una autopsia médicolegal sus deudos no acreditan su condición de tales ni expresan su oposición en el mismo sentido" 30 . Es decir, que la presunción admitía prueba en contrario, situación que en la actualidad no es plausible, pues según el artículo 3, "se presume que se es donante cuando una persona durante su vida se ha abstenido de ejercer el derecho que tiene a oponerse a que de su cuerpo se extraigan órganos, tejidos o componentes anatómicos después de su fallecimiento" ${ }^{1}$.

29 Dice el artículo 2 de la Ley 1805 del 2016: "Solo se podrá proceder a la utilización de los órganos, tejidos, componentes anatómicos y líquidos orgánicos a que se refiere este artículo, cuando exista consentimiento del donante libre, previo e informado o presunción legal de donación”.

30 Al respecto, establecia el artículo 6 del Decreto 1546 de 1998 que "para ejercer el derecho a oponerse, los deudos deberán presentarse y acreditar dicha condición, dentro del lapso de seis horas siguientes a la ocurrencia de la muerte encefálica o antes de la iniciación de una necropsia, y expresar su oposición, en caso de no haberse expresado la voluntad de donar en vida” (Tirado Acero, 2011).

31 Rojas-Delgado, Gutiérrez-Montaña, Rojas Rivera, Saavedra Ávila y Nancupil Reyes (2014) aducen que el sistema de distribucción de órganos, mediante el criterio de priorización de la donación recíprocra, genera inequidades asimétricas. 
Esta manifestación de la presunción legal de donación ${ }^{32}$, según el artículo 4 de la Ley 1805, debe celebrarse mediante un documento escrito en el cual se establezca la voluntad de no ser donante de órganos ni tejidos; debe autenticarse, además, ante notario público y radicarse en el Instituto Nacional de Salud (INS). No obstante, la manifestación podrá expresarse al momento de afiliarse a una empresa prestadora de salud (EPS), que tiene la obligación de reportarla al INS.

\section{DisCuSión DE RESUlTADOS}

Con respecto a los criterios para priorizar a los pacientes y asignar los órganos en el proceso de trasplante, resaltó la Corte Constitucional a la luz de la legislación anterior, que

no existen criterios definidos para la adjudicación de los órganos disponibles. La regulación simplemente determina en el artículo 25 del Decreto 2493 de 2004, el principio de la no discriminación, así como la distribución territorial de los órganos y el artículo 29 de la Resolución 2640 de 2005, que tan solo avala los criterios de asignación de componentes anatómicos, es decir, aquellos empleados por las instituciones prestadoras de servicios de salud hasta el momento. (Córdoba Triviño, 2006)

La Ley 1805, por el contrario, parece haber optado por un sistema opt-out ${ }^{33}$ como criterio básico para establecer prioridades porque en su artículo 14 establece que "en

32 Según la línea jurisprudencial de la Corte Constitucional, esta imposición de la imposiblidad de presentar oposición por parte de los familaires resultaría en contravía de la interpretación constitucional vigente. Esto ocurre porque "advierte la Corporación que la presunción de donación no puede cercenar el derecho de los familiares a negarse a la ablación de órganos y componentes anatómicos del cadáver de su ser querido. Negación que se pueda dar por el rango constitucional de la libertad individual y los derechos de libertad de conciencia, de religión y de cultos de los familiares. 'El Estado debe asumir frente al tema de la donación de órganos una posición neutra e imparcial respetando las diferentes ideologías o concepciones sobre el bien y lo bueno de los ciudadanos"” (Carreño Dueńas et al., 2016).

33 Este sistema ofrece un doble incentivo para la donación: por un lado evita los costos de registrarse como donante, los traslada a los no donantes y, por el otro, genera una priorización en la lista de espera. Evita en el proceso que los free-rides se beneficien, salvo que el sistema haya generado suficientes órganos como para suplir tambien la demanda de estos (Zúñiga-Fajuri, 2015). 
aquellos casos en los cuales dos (2) personas en lista de espera de trasplante de órganos o tejidos sean médicamente compatibles y tengan el mismo nivel de gravedad, el órgano o tejido será trasplantado a la persona que hizo expresa su voluntad de ser donante de órganos y tejidos y se encuentre identificada como tal". Al respecto, debe mencionarse que este no es más que un trasplante normativo en el cual se combina la prioridad con la donación presunta como mecanismos para elevar los índices de órganos disponibles para trasplantes ${ }^{34}$.

Esta norma tiene su origen en la Ley de trasplante de órganos humanos de Singapur de 1987, que establece que si existiera un conflicto por un órgano para trasplante entre dos personas, este se debe entregar a quien hubiese manifestado su voluntad de ser donante al momento de su muerte (Zúñiga-Fajuri, 2015; Iyer, 1987). Este modelo fue después implementado en Israel en el 2008, mediante la Ley de trasplante de órganos ${ }^{35}$ y en Chile, en el 2010, mediante la Ley $20.413^{36}$, así:

El sistema es consistente con la idea de que, ya que la oferta de donantes de órganos ha sido altamente superada por la demanda y que estamos ante lo que podemos calificar como un "recurso social muy escaso", los principios de justicia distributiva demandan la incorporación de una regla de altruismo

34 Afirma el Dr. Eduardo Santiago-Delpín que, en un sistema de donación presunta, "los órganos de cadáveres pueden recuperarse sin un consentimiento explícito, a menos que el difunto hubiera hecho alguna objeción al respecto en vida. Existen dos variedades de este sistema: (1) débil: cuando los familiares pueden evitar la adquisición de órganos negándose a la donación; y (2) fuerte: cuando la opinión de los familiares no se toma en cuenta" (Santiago-Delpín et al., 2001, p. 80, citados por Carreño et al., 2016, p. 71 ).

35 "En enero del 2010, la Organ Transplant Act 2008, que regula la donación y adjudicación de órganos, entró en vigencia en Israel estableciendo un sistema de puntos que da carácter preferencial para recibir un órgano a quienes están dispuestos a ser donantes. Como resultado, el sistema establece una tabla escalonada que incluye la máxima prioridad, prioridad regular y segunda prioridad. La máxima prioridad se otorga a: (a) quienes han firmado el consentimiento de donación o a los familiares directos de personas que han donado sus órganos (b) y a quienes han donado en vida un riñón o hígado a un receptor no especificado. La prioridad regular la tienen aquellos candidatos que poseen una tarjeta de donantes. Por último, la segunda prioridad se da a quienes, sin poseer la tarjeta de donante, tienen un familiar directo que sí ha manifestado su intención de donar al momento de su muerte. "Desde la vigencia de la ley, Israel ha sido testigo de un número récord de firmas de la tarjeta de donante y se ha producido un aumento significativo en el número real de trasplantes" (Zúńiga, 2015, p. 1332).

36 Esta no solo establece la donación presunta y un sistema de priorización basado en el opt-out, sino que también obliga a las autoridades a mantener un registro de no donantes, tal como sucede en Colombia, como resultado de lo establecido en la Ley 1805 del 2016. 
recíproco. Ello permite corregir la injusticia derivada de los llamados free riders, aquellas personas que utilizan recursos sociales limitados eludiendo aportar al costo de su producción. La voluntad de ser donante de órganos o tejidos a cambio de ganar la calidad de "elegible" para la recepción de los mismos, parece un requisito moral básico de justicia. (Congreso Nacional de Chile, 2010)

Zúńiga (2015) dice que existe un deber moral de reciprocidad ${ }^{37}$ generalmente llamado "deber de ayuda mutua" o "deber de socorro", que

ha sido el fundamento de las leyes promulgadas en diferentes países que obligan a todos los ciudadanos (no solo médicos o policías) a ayudar a una persona en grave peligro, en la medida en que ello no signifique un costo excesivo o un riesgo real para el socorrista (Peters, 1988-1989). Con el consentimiento presunto los órganos para trasplante, una vez que han sido donados, se transforman en un bien público pues es el Estado el que regula su adjudicación. A su turno, todas las personas - como ocurre con los bienes públicos - tienen derecho a ellos cuando los necesitan, incluso los free riders, pues la norma chilena establece una regla de prioridad para el trasplante, no de exclusión. Jarvis sostiene que "aquellos y solo aquellos que eligen potencialmente contribuir al sistema, están en posición de beneficiarse de él” (Jarvis, 1995). Ello permitiría disminuir la demanda de órganos — pues se excluiría a quienes han optado por no sumarse al esquema de donación-, aumentaría el número de donantes — pues claramente la gente preferirá formar parte del sistema y, finalmente como beneficio marginal, se promovería la solidaridad y el altruismo en vez del egoísmo. (p. 1332)

Con respecto a la donación de órganos y sus implicaciones, ha reconocido la Corte Constitucional la existencia de un conflicto de derechos fundamentales (Patarroyo

37 "La vieja teoría del contrato social se sustenta en la premisa de que es posible derivar un beneficio social mayor cuando la gente actúa en su propio interés. Es decir, bajo un esquema en el que cada cual decide sobre la base de aquello que más le conviene, se actúa también en el interés de toda la comunidad promoviéndose el bien común. Además, la regla de la reciprocidad permite recibir los beneficios mientras se está vivo imponiendo los costos solo una vez que hemos muerto lo cual, finalmente, no solo es justo, sino que pareciera ser un buen trato" [énfasis ańadido] (Zúñiga, 2015, p. 1335). 
y Benavides, 2014; Humbarita, 2015) en el que está, por un lado, el derecho a la vida de quien espera un trasplante y, por el otro, los derechos de los herederos y familiares de quien presenta muerte encefálica ${ }^{38}$. En lo referente a los derechos de estos últimos, ha dicho la Corte Constitucional en Sentencia C-933 del 2007:

Respecto a la cláusula general de libertad, y los derechos a la libertad de conciencia, de religión y de culto que cabe reconocerle y protegerle a los familiares de una persona fallecida, y que están asociados a la posibilidad de disponer y rendirle culto a los muertos en el cuerpo de la persona querida ya $\sin$ vida $^{39}$.

No obstante, esta tensión entre derechos fundamentales (Quiroz, 2014) resulta mínima cuando se analiza a la luz de los principios constitucionales, particularmente el principio de solidaridad como principio fundamental de la creación del Estado ${ }^{40}$, porque su aplicación conllevaba la determinación de una donación necesaria en el marco del cumplimiento de los deberes del ciudadano, lo que se debe a que el principio de solidaridad

supone la colaboración de la sociedad con aquellas personas situadas en una posición de desventaja (CP, art. 1) Sentencia C-529/10. Las implicaciones de la concreción de este principio son acciones en atención del beneficio de los otros, como imperativo del interés general, como lo propio en un Estado social de derecho, que configura una comunidad de intereses, sentimientos y aspiraciones: de la cual emana, como consecuencia natural y obvia, un acuerdo

38 Entre los que se deben contar la libertad de consciencia, la libertad de cultos y el libre desarrollo de la personalidad, así como el derecho a la intimidad personal y familiar.

39 "Para la Alta Corporación, este derecho a la disposición del cadáver, está fundamentado en derechos esenciales como la libertad de conciencia, de religión y de cultos, como así lo ha expresado en sentencias como la T-162 de 1994 y la T- 462 de 1998" (Carreño et al., 2016).

$40 \mathrm{Al}$ respecto, señalan Carreño et al. (2016) frente a este proceso de ponderación: "La Corte asegura al principio de proporcionalidad toda la fuerza de razonabilidad exigida, como quiera que debe en aras de la dimensión del peso o de la importancia, sopesar un principio frente a otro principio, y debe prever la decisión más adecuada o correcta a los fines de un Estado social de derecho: [...] declarar inválido al principio desplazado ni que en el principio desplazado haya que introducir una cláusula de excepción [...] bajo ciertas circunstancias, uno de los principios precede al otro [...] las colisiones de principios — como quiera que solo pueden entrar en colisión principios válidos - tienen lugar más allá de la dimensión de la validez, en la dimensión de peso” (Alexy, 2008, p.71). 
de mutua ayuda y una responsabilidad compartida para el cumplimiento de los fines propuestos: la satisfacción de las necesidades individuales y colectivas. (Sentencia C-32 del 2008)

Igualmente, la Corte Constitucional ha cerrado jurisprudencialmente la oportunidad de obtener por vía de tutela la realización de un trasplante; en términos perentorios, evita la espera del sistema de turnos, de ahí que con esta acción se afectarían los derechos de otros pacientes que, al estar en la misma situación, se verían desplazados de su lugar en la lista. Ello constituye un despropósito infundado en la Constitución, pues esta no solo es constitucionalmente legítima a la luz de sus objetivos, sino que no vulnera en forma alguna el derecho a la salud (Córdoba Triviño, 2006) ${ }^{41}$.

Finalmente, al analizar la reforma normativa en materia de publicidad para incentivar la donación de órganos por parte de la Ley 1805 de 2016 (se invertirá el 15\% del presupuesto para pautas de las entidades prestadoras de salud en promocionar el alcance de la norma), se encuentra que esta es ineficiente porque el establecimiento de una presunción de consentimiento, lejos de fomentar las campañas, acaba con ellas, al hacerse innecesaria la manifestación de voluntad. Según Rabinovich (2007), conllevaría la pérdida de cuanto (el trasplante) "tiene de magnífico, de noble, de humano, para pasar a ser una mera estadística tecnócrata, materialista y hasta truculenta”.

\section{Conclusiones}

La modificación del marco regulatorio de la donación de órganos, introducida mediante la Ley 1805 del 2016, instauró tanto el establecimiento de una presunción legal de donación como un polémico criterio de priorización para asignar órganos en caso de que dos pacientes de la misma gravedad estén a la espera de recibir un mismo órgano. Esta reforma normativa resulta conflictiva, no solo con las concepciones

41 Mediante el Decreto 2493 del 2004 y la Resolución 2640 del 2005, se estableció la organización de la Red de Trasplantes en dos niveles: el nacional a cargo del Instituto Nacional de Salud y el regional, en las secretarías departamentales y distritales de salud. Los turnos para trasplante son asignados por cada una de las iPs según el marco regulatorio del artículo 7, numeral 3, del Decreto 2493 del 2004 y el artículo 7 de la Resolución 2640 del 2005 (González Cuervo, T-111 de 2010). 
sociales, sino con los derechos individuales y colectivos de los donantes y sus familiares, porque, al no admitir prueba en contrario - por ejemplo, en el caso de algunas religiones-, se vulnera el carácter sagrado del cuerpo y el derecho a la libertad de cultos de la familia, que estaría en la imposibilidad de resistirse a la donación, lo que se torna en una afrenta a su sistema de creencias.

Es igualmente perjudicial el establecimiento de una presunción de donación de órganos porque retira de la ecuación los componentes humano y moral de la acción misma y desnaturaliza el proceso de la donación al evitar la concreción de su componente altruista, ampliamente considerado como elemento esencial por las diversas religiones. Lo anterior se constata con la existencia del "incentivo" establecido en la Ley 1805 que conlleva un incremento en los niveles de donación, pero no por la voluntad de las personas de donar, sino por la falta de alternativas u obligatoriedad que implica la priorización de quienes donan, frente a quienes no donan tras su muerte, para la asignación de órganos, bien sea para sí mismos o para sus familiares cercanos.

Existen otras alternativas, como política pública, de solución al desequilibrio entre oferta y demanda de donación de órganos, como son la implementación de programas educativos y de concientización de alto impacto o el establecimientos de mecanismos simples para otorgar el consentimiento de la donación. Este modelo es implementado en algunos países con una pregunta simple: ¿̇desea ser donante de órganos?, al momento de tramitar la cédula de ciudadanía, la licencia de conducción o el pasaporte. Con ello se garantiza la donación, se preserva el componente altruista, se respetan los derechos de los donantes y se evita la vulneración de terceros al disponer del cuerpo sin considerar el sistema de creencias que el difunto haya tenido en vida.

La política normativa establecida mediante la Ley 1805 del 2016 resulta eficiente en términos económico-jurídicos para solucionar el desequilibrio existente entre oferta y demanda de donación de órganos, así como asegurar mecanismos para garantizar el derecho fundamental a la vida digna - presupuesto esencial para el ejercicio de cualquier derecho-. Pese a esto, también resulta ineficiente e irracional en términos sociojurídicos porque genera espacios que vulneran derechos de terceros, en detrimento del sistema mediante el cual estos individuos construyen su percepción de la realidad social. 
En cuanto a la racionalidad jurídico-económica y social de la donación de órganos presunta legalmente, establecida en el artículo 3 de la Ley 1805 del 2016, esta norma es racional en términos jurídico-económicos porque corrige, al menos de forma teóri$\mathrm{ca}$, el desequilibro entre donantes y receptores de órganos. Al mismo tiempo, ampara en el proceso derechos fundamentales (en detrimento de otros de menor relevancia) y prolonga de forma digna la vida de quien recibe un órgano, con lo cual se cumple con los postulados constitucionales. No obstante, no deja de ser problemática la imposición sobre el cuerpo realizada mediante esta presunción porque es posible afirmar que existen mecanismos idóneos para solucionar el desequilibrio entre oferta y demanda de órganos, sin que se vulneren en el proceso derechos de terceros o del cadáver.

Finalmente, en lo que se refiere a la asignación de recursos para promocionar la donación de órganos, sus implicaciones, características y particularidades deberán ser analizadas por futuros estudios que tengan en cuenta el impacto de las medidas actuales con el fin de determinar la eficacia y la eficiencia de la norma en cuestión, así como la racionalidad de la inversión.

\section{REFERENCIAS}

Alexy, R. (2008). Teoría de los Derechos Fundamentales. Madrid: Centro de Estudios Politicos y Constitucionales.

Alonso-Pulpón, L., Almenar, J., Crespo, M. G., Silva, L., Segovia, J., Manito, N. et al. (1995). Guías de actuación clínica de la sociedad española de cardiología. Transplante cardiaco y de corazón-pulmones. Rev. Esp. Cardiol., 52(10), 821-839.

Álvarez Hidalgo, R. (mayo-agosto del 2004). El criterio inmunológico de selección para el transplante renal: Un aspecto científico-ético. Humanidades Médicas, 4(2), 1-13.

Álvarez, H. (2007). Consentimineto presunto y respuesta requerida como alternativa a la escasez de donantes: un análisis ético. Revista Chilena de Cirugía, 59, 244-249.

Barrera Sánchez, Ó. (2011). El cuerpo en Marx, Bourdieu y Foucault. Iberoforum, 121-137.

Barriga, C., Baeza, R., Pereira, J., Besa, P., Caldumbide, I. y Medel, M. (1999). Transplante de médula ósea en pacientes pediátricos. Rev. chil. Pediatr., 70(3), 194-200. 
Bourdieu, P. (1986). Notas provisionales sobre la percepción social del cuerpo. En Materiales de Sociología Crítica. Madrid: La Piqueta.

Bourdieu, P. (1991). El sentido práctico. Madrid: Taurus.

Bustamante, M. y Villareal, P. (2009). Los medios de comunicación en el proceso de donación de órganos en Chile. Panorama Socioeconómico, 27(38), 60-67.

Caballer Miedes, A. y Martínez Sancho, M. (2000). Variables influyentes en la actitud hacia la donación de órganos. Psicothema, 12(Su. 2), 100-102.

Cantarovich, F. (2004). The role of education in increasing organ donation. Ann Transplantation, 9, 39-42.

Carreño Dueñas, D., Restrepo Restrepo, J. A. y Becerra, J. (2016). Aspectos sociojurídicos de la donación y transplante de órganos en Colombia. En D. Carreño Dueñas, Y. M. Guerra, J. A. Restrepo Restrepo, J. Becerra, M. Tirado Acero, J. C. Bedoya Chavarriaga et al. Bioética y derecho (pp. 55/90) (78). Bogotá: Ibáńez- Universidad Santo Tomás.

Castro, E. (1995). Pensar a Foucault: interrogantes filosóficos de la arqueología del saber. Buenos Aires: Biblos.

Colombia. Corte Constitucional. (1994). Sentencia T-067, T-24508. M. P. José Gregorio Hernández Galindo.

Colombia. Corte Constitucional. (1994). Sentencia T-162, T-28107. M. P. Eduardo Cifuentes Muñoz.

Colombia. Corte Constitucional. (1998). Sentencia T 645, T- 180262. M.P Fabio Morón Díaz.

Colombia. Corte Constitucional. (2006). Sentencia T-568, T-1113653. M. P. Jaime Córdoba Triviño.

Colombia. Corte Constitucional. (2007). Sentencia T-933 de 2007. M. P. Jaime Araujo Rentería.

Colombia. Corte Constitucional. (2008). Sentencia C-32. M. P. Marco Gerardo Monroy Cabra.

Colombia. Corte Constitucional. (2010). Sentencia T-111, T-2.360.513. M. P. Mauricio González Cuervo. 
Colombia. Corte Constitucional. (2012). Sentencia T-675, T-3.420.766. M.S. Mauricio González Cuervo.

Colombia. Congreso de la República. Ley 9 (1979). Diario Oficial No. 35308. "por la cual se dictan Medidas Sanitarias".

Colombia. Congreso de la República. Ley 73 (1998). Diario Oficial No. 38.623 "Por la cual se adiciona la Ley 09 de 1979 y se dictan otras disposiciones en materia de donación y trasplante de órganos y componentes anatómicos para fines de transplantes $\mathrm{u}$ otros usos terapéuticos”.

Colombia. Ministerio de Salud. Decreto 1546 (1998). Diario Oficial No. 43.357. "Por el cual se reglamentan parcialmente las Leyes 9a. de 1979, y 73 de 1988, en cuanto a la obtención, donación, preservación, almacenamiento, transporte, destino y disposición final de componentes anatómicos y los procedimientos para trasplante de los mismos en seres humanos, y se adoptan las condiciones mínimas para el funcionamiento de las Unidades de Biomedicina Reproductiva, Centros o similares”.

Colombia. Ministerio de la Protección Social. Decreto 2493 (2004). Diario Oficial No. 45.631. "Por el cual se reglamentan parcialmente las Leyes 9a de 1979 y 73 de 1988, en relación con los componentes anatómicos”.

Colombia. Ministerio de la Protección Social. Resolución 2640 (2005). Diario Oficial No. 46.007. "por medio de la cual se reglamentan los artículos $3^{\circ}, 4^{\circ}, 6^{\circ}$ parágrafo $2^{\circ}, 7^{\circ}$ numeral 10, 25 y 46 del Decreto 2493 de 2004 y se dictan otras disposiciones. El Ministro de la Protección Social, en ejercicio de sus facultades legales, en especial, las conferidas por el Decreto 205 de 2003 y el Decreto 2493 de 2004”.

Colombia. Congreso de la República. Ley 1805 (2016). Diario Oficial No. 49.955. "Por medio de la cual se modifican la Ley 73 de 1988 y la Ley 919 de 2004 en materia de donación de componentes anatómicos y se dictan otras disposiciones”.

Chile. Congreso Nacional. Ley 20.413. (2010). La cual “Modifica la ley n 19.451, con el fin de determinar quiénes pueden ser considerados donantes de órganos y la forma en que pueden manifestar su voluntad".

Cortés, S. (enero-junio del 2016). Derechos humanos en las políticas de paz y posconflicto en Colombia. Via Inveniendi et Iudicandi, 11(1), 129-145. Recuperado de http://revistas. usantotomas.edu.co/index.php/viei/article/view/2926/2798 
Demp, B. (2014). The quotable Coach: Daily nuggets of practical wisdom. Seattle: CreateSpace Independent Publishing Platform.

Erdogan, O., Yucetin, L., Tuncer, M., kececioglu, N., Gürkan, A., Akaydin, M. et al. (2002). Attitudes and knowledge of Turkish physicians about organ donation and transplantation. Transplant. Proc., 34, 2007-2008.

Farfán, F. (2006). Tráfico de órganos humanos y ley penal. Bogotá: Procuraduría General de la Nación.

Feuerbach, L. (1881). The Essence of Christianity. London: Trübner \& Co.

Foucault, M. (1975). Surveiller et punit: naissance de la prisión. París: Gallimard.

Foucault, M. (1976). Histoire de la sexualité. París: Gallimard.

Foucault, M. (1990). Genealogía del racismo. Buenos Aires: Altamira .

Foucault, M. (1992). Microfísica del poder. Madrid : La Piqueta.

Freitas, N. K. (2008). Body schemes, visual images and representations of self body: theoretical and conceptual questions. Ciênc Cogn, 13(1), 318-324.

Giddens, A. (2000). Sociología. México: Alianza.

Gómez-Rázuri, K., Ballena-López, J. y León-Jiménez, F. (2016). Mitos sobre la donación de órganos en personal de salud, potenciales receptores y familiares de potenciales donantes en un hospital peruano: estudio cualitativo. Revista Peruana de Medicina Experimental y Salud Pública, 33(1), 83-91.

Gracia, D. (2001). Transplante de órganos: medio siglo de reflexión ética. Nefrología, 21(supl 4), 13-29.

Grudka Liral, G., Pontes, C. M., Schirmer, J. y Soares, L. (2012). Family considerations about the decision to refuse organ donation. Acta Paulista de Enfermagen, 25(spe. 2), $140-145$.

Guerra, Y., Tirado, M., Márquez, A. (2012) Trasplante de órganos, bioética y legislación comparada. Bogotá: Universidad Militar Nueva Granada, Editorial Periódicas.

Humbarita, J. (2015). Derecho constitucional hispanoamericano frente a la realidad institucional: manifiesta divergencia. Revista IUSTA, 43(2). 
Instituto Nacional de Salud: Red de Transplantes (19 de octubre del 2016). Instituto Nacional de Salud. Informe Ejecutivo Red Donación y Transplantes 2015: Recuperado de http://www.ins.gov.co/lineas-de-accion/Red-Nacional-Laboratorios/Estadsticas/ Informe\%20Ejecutivo\%20Red\%20Donación\%20y\%20Trasplantes\%20\%202015. pdf? Mobile $=1 \&$ Source $=\% 2$ Flineas - de-accion $\% 2$ FRed-Nacional-Laboratorios $\% 2 F_{-}$ layouts\%2Fmobile\%2Fview.aspx\%3FList\%3Daa64

Iyer, T. K. (1987). Kidneys for transplant. "Opting out" law in Singapore. Forensic Sci Int., 131-140.

Jarvis, R. (1995). Join the club: a modest proposal to increase availability of donor organs. $J$ Med Ethics, 21(4), 199-204.

Jung, H. (2013). Reluctance to donate organs: a survey among medical students. Transplant. Proc., 45, 1303-1304.

Koury, M., Scribeno, A. y Tijoux, M. E. (2011). Acta Cientifica. El cuerpo en la sociología. Chile: Memorias del XXIX Congreso de ALAS.

Kovacs, M. J. (2003). Bioethics concerning life and death. Psicol. USP., 14(2), 115-167.

Le Breton, D. (2008). La sociología del cuerpo. Nueva Visión: Buenos Aires .

Lehmann. (1949). Allgemeiner Teil des BGD, parte general. Berlín.

Marx, K. (s.f.). Tesis sobre Feuerbach. Madrid: Luarna Ediciones.

Marx, K. (1976). Manuscritos económico-filosóficos de 1884. México: Cultura Popular.

Marx, K. (1991). El Capital. Crítica de la economía política. México: Siglo xxI.

Mateasanz, R. y Miranda, B. (2002). A decade of continuous improvement in cadaveric organ donation: the spanish model. Journal Nephrol., 15, 22-28.

Mauss, M. (1979). Sociología y antropología. Madrid: Tecnos.

Ministerio de Salud. Dirección de Medicamentos y Tecnologías en Salud (24 de marzo del 2015). Datos globales de donación y transplantes, 2014. Recuperado de https://www. minsalud.gov.co/sites/rid/Lists/BibliotecaDigital/RIDE/VS/MET/Datos-Globales-Donacion-Trasplantes.pdf 
Ministerio de Salud y Protección Social (27 de octubre del 2016). Donaciones y transplantes de órganos. Por eso déjalo conversado. Recuperado de https://www.minsalud.gov.co/salud/ MT/Paginas/donacion-y-trasplantes-de-organos.aspx

Moloney , G. y Walker, I. (2000). Messiahs, pariahs, and donors: the development of social representations of organ transplants. J Theory Soc Behav, 203-227.

Moloney, G. y Walker, L. (2002). Talking about transplants: social representation and the dialetical, dilemmatic nature of organ donation and transplantation. Brj. J. Soc. Psychol., 41(2), 299-320.

Moreno-treviño, M. G. y Rivera-Silva, G. (2015). Donación de órganos, tejidos y células en México. Revista Médica del Instituto Mexicano del Seguro Social, 53(6), 762-763.

Moscovici, S. (2007). Representacoes sociais: investigacoes em psicologia social. Petrópolis: Vozes.

Namihira, E. (1990). Shinto concept concerning the dead human body. Transplant Proc, $22,940-941$.

Oliveira, R. A. y Oselka, G. (2008). Terminalidadde da vida: doenca incurável e morte encefálica. En M. J. Kovács y I. Esslinger, Dilemas éticos (pp. 81-89). São Paulo: Centro Universitário São Camilo.

Padilla-Cuadra, J. I., Mora-Chacón, P., Monge-Fallas, A. y Rodríguez-Barquero, R. (2015). Actitudes y conocimientos sobre la donación de órganos, transplantes y muerte cerebral en estudiantes de ciencias de la salud. Acta Médica Costarricense, 57(4), 179-183.

Palacios, J. M. (2002). Procuramiento de órganos: el modelo chileno. Revista Chilena de Cirugia, 54, 573-588.

Patarroyo, S. y Benavides, P. (2014). Rupturas asignificantes: revisiones críticas en torno al derecho. Via Inveniendi et Iudicandi, 9( 1), 7-31.

Peters, D. A. (1988-1989). A unified approach to organ donor recruitment, organ procurement, and distribution. J Law Health, 3(2), 157-187.

Posner, R. (2013). Análisis económico del derecho. Madrid: Fondo de Cultura Económica.

Prieto, M., Clemente, G., Casafont, F., Cuende, N., Cuervas-Mons, V., Figueras, J. et al. (2003). Documento de consenso de indicaciones de transplante hepático. Gastroenterol. Hepatol., 26(6), 355-375. 
Quiroz, M. (2014). Acercamiento a las “oposiciones paradigmáticas” entre neoconstitucionalismo y positivismo jurídico. Revista IUSTA, 41(2), 77-97.

Rabinovich-Berkman, R. (2007). Transplante de órganos y tejidos. Buenos Aires: Astrea.

Reddy, A. V., Guleri, S., Khazanchi, R. K. et al. (2003). Attitude of patients, the public, doctors, and nurses toward organ donation. Transplant Proc, 35, 18.

Reyes Acevedo, R. (2005). Ética y transplantes de órganos: búsqueda contínua de lo que es aceptable. Revista de Investigación Clinica, 57(2), 177-186.

Ríos, A., Conesa, C., Ramírez, P., Galindo, P. J., Rodríguez , J. M., Rodríguez, M. M., et al. (2006). Attitudes of resident doctors toward differente types of organ donation in a Spanish transplant hospital. Transplant. Proc., 38, 869-874.

Rojas-Delgado, P., Gutierrez-Montańa, M., Rojas Rivera, J., saavedra Avila, L. y Nancupil Reyes, W. (2014). Una mirada a la nueva ley de donación de órganos en Chile: la polémica prioridad para los donantes. Revista de Facultad de Medicina, 62(3), 477-480.

Roza, B. A., García, V. D., Barbosa, S. F., Mendes, K. D. y Schirmer, J. (2010). Organ and tissues donation: relation with the body in our society. Acta Paulista de Enfermagem, 23(3), 417-422.

Rumsey, S., Hurdford, D. P., Cole, A. K., Rojas, A. y Serani, A. (2003). Influence of knowledge and religiousness on attitudes toward organ donation. Transplant proc, 35, 2845-2850.

Sabido, O. (2011). El cuerpo y la afectividad como objetos de estudio en América Latina: intereses temáticos y proceso de institucionalización reciente. Sociológica, 26(74), 33-78.

Salvat, M. (1975). La sociedad del consumo. Barcelona: Salvat.

Santos, M. F. y Almeida, L. M. (2007). Diálogos com a teoria da representacäo social. Petrópolis: Vozes.

Sheehy, E., Conrad, S. L., Brigham, L. E., Luskin, R., Weber, P., Eakin, M. et al. (2003). Estimating the number of potential organ donors in the united states. New England J. Med., 349, 667-674.

Santiago-Delpín et al. (2001). Trasplante, ética, humanismo y sociedad. México, Bogotá: JGH Editores. 
Sodi, L. (2003). La experiencia de donar: estudio antropológico sobre la donación de órganos. México: Plaza y Valdés.

Solar, S., Ovalle, A., Simian, M. E., Escobar, J. y Beca, J. P. (Junio del 2008). Tres factores que influyen en la actitud de las personas ante la donación de órganos. Revista Chilena de Cirugia, 60(3), 262-267.

Sossa Rojas, A. (2012). Análisis desde Michel Foucault referentes al cuerpo, la belleza fisica y el consumo. Polis, 28, 1-19.

Sugunasiri, S. (1990). The buddhist view concerning the dead body. Transplant Proc, 22, 947-949.

Tirado Acero, M. (2011). ¿Por qué en Colombia no está generalizada la práctica de la donación de órganos? Revista Latinoamericana de Bioetica, 11(1), 56-67.

Tirado Acero, M. (2011a). Aproximación desde la Sociología Jurídica a Dilemas de la Bioética Contemporánea: "Muertos Con Corazón Viviente". Revista Brasilera de Bioetica, $7(1-4) \cdot 6-21$

Valencia Zea, A. y Ortiz Monsalve, Á. (2011). Derecho civil: parte general y personas (16. ${ }^{\mathrm{e}}$ ed., vol. I). Bogotá: Temis.

Weber, F. y Canbay, A. E. (1999). Attitudes of physicians and nursing staff member toward organ donation in an urban area of Germany. Transplant. Proc., 31, 2179-2180.

Weber, M. (2012). Sociología de la religión. Valencia: Akal.

Zúñiga-Fajuri, A. (2015). Increasing organ donation by presumed consent and allocation priority: Chile. Bulletin of the World Health Organization, 199-202.

Zúniga, A. (2015). El consentimiento presunto y la reciprocidad como mecanismos para aumentar la donación de órganos. Revista Médica de Chile, 143(10), 1331-1336.

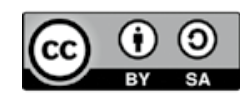

